\title{
Small-Angle X-Ray Scattering Studies of Pore Structure in Cellulose Membranes
}

\author{
Cz. ŚlumsarczyK*, B. Fryczkowska, M. Sieradzka and J. Janicki \\ Institute of Textile Engineering and Polymer Materials, University of Bielsko-Biała, \\ Willowa 2, 43-309 Bielsko-Biała, Poland
}

\begin{abstract}
Small-angle X-ray scattering experiments were carried out on cellulose membranes obtained by phase inversion from a solution of the polymer in 1-ethyl-3-methylimidazolium acetate, by coagulation in water and selected primary alcohols. The invariant, $Q$, and the Guinier radius, $R_{\mathrm{G}}$, computed directly from the small-angle X-ray scattering curve allowed us to estimate the pore dimensions and the pore volume fraction. It was found that both content and dimensions of pores depend on molecular mass of the coagulant used. Also, it was found that the dipole moment of coagulant molecules has a large influence on the volume content of the pores.
\end{abstract}

DOI: 10.12693 /APhysPolA.129.229

PACS: 61.05.cf, 68.55.J-, 82.35.Pq

\section{Introduction}

Among the natural organic compounds cellulose is seen as the most widespread, inexpensive, biodegradable polymer derived from renewable sources [1], which is widely used in various industries $[2,3]$. Cellulose is a linear polymer linked with stable glycosidic bonds, accompanied by intra- and intermolecular hydrogen bonds which makes this polymer insoluble in water and in most organic solvents $[2,4,5]$. No solubility poses serious limitations in cellulose processing technology.

An interesting group of new solvents are ionic liquids, often referred to as "green" solvents. Due to the biodegradability [6] and low toxicity [7] they are considered to be environmentally friendly solvents, which may eventually replace the traditional systems that are capable of dissolving cellulose $[8,9]$. Cellulose dissolved in ionic liquids can be easily coagulated, using polar solvents such as water, acetone, ethanol, dichloromethane, acetonitrile, or by using a mixture of these solvents $[10,11]$. The process of precipitation and selection of the coagulant affects the coagulated polymer structure.

Among products made from regenerated cellulose, which deserve special attention, are certainly membranes. These materials are widely used in a variety of membrane separation techniques, such as dialysis, ultrafiltration, fraction and purification of mixtures, which are used in sewage treatment plants in the process of water treatment, in the chemical and food industry, as well as in environmental protection, biochemistry, and medicine $[12,13]$. According to the literature reports, the factors affecting the properties of membranes include, among others, the type of coagulating bath, its concentration, and the time of film formation [12].

*corresponding author; e-mail: cslusarczyk@ath.bielsko.pl
This paper presents an analysis of the porous cellulose membranes structure using the small angle X-ray scattering (SAXS) method. Using this method, the pore dimensions and the pore volume fraction have been determined. The studies characterize pore sizes on length scales from $1 \mathrm{~nm}$ to $60 \mathrm{~nm}$, according to the resolution of the SAXS equipment used.

\section{Experimental}

\subsection{Preparation of a cellulose solution and membranes}

A $5 \%$ solution of the long-fiber cellulose in the ionic liquid - 1-ethyl-3-methylimidazolium acetate - was prepared. To do this, appropriate amounts of cellulose and ionic liquid were weighed, everything was mixed well and then heated in a microwave oven until clear solution was obtained. The solutions were allowed to deaerate for $24 \mathrm{~h}$.

Cellulose membranes were prepared using phase inversion method. For this purpose, a $5 \%$ solution of the cellulose in the ionic liquid was poured onto the leveled, clean glass plate forming a polymer film using membrane applicator with a gap width of $0.2 \mathrm{~mm}(200 \mu \mathrm{m})$.

Then the plate with a spread cellulose solution was transferred to a coagulant bath. Water and the selected primary alcohols, listed in Table I, were used for the coagulation of the membranes. The membranes obtained in this way were placed between the layers of filter paper, pressed and left to dry.

\subsection{SAXS measurements}

The small-angle X-ray scattering (SAXS) measurements were carried out with the compact Kratky camera, equipped with the SWAXS optical system of HECUSMBRAUN (Austria). The $\mathrm{Cu}$ target $\mathrm{X}$-ray tube, operated at: $U=30 \mathrm{kV}, I=10 \mathrm{~mA}$ was used as a radiation source $(\lambda=0.154 \mathrm{~nm})$. The SAXS data were collected as a function of the scattering vector $q=(4 \pi / \lambda) \sin \theta$, where $2 \theta$ is the scattering angle. The moving slit method [14] was applied for determination of the transmittance factor of the sample. The sample holder background was 
TABLE I

Characteristics of the samples used for the study and values of structural parameters obtained by means of SAXS method.

\begin{tabular}{c|c|c|c|c|c}
\hline \hline \multirow{3}{*}{ Sample } & \multicolumn{3}{|c|}{ Coagulant } & \multicolumn{2}{c}{ SAXS results } \\
\cline { 2 - 6 } & Type & $\begin{array}{c}\text { Molar } \\
\text { mass } \\
{[\mathrm{g} / \mathrm{mol}]}\end{array}$ & $\begin{array}{c}\text { Dipole } \\
\text { moment } \\
{[\mathrm{D}]}\end{array}$ & $\begin{array}{c}R_{\mathrm{G}} \\
{[\mathrm{nm}]}\end{array}$ & $\Phi$ \\
\hline$M$ & methanol & 32.04 & 1.70 & $11.0 \pm 0.3$ & 0.71 \\
$E$ & ethanol & 46.07 & 1.69 & $12.2 \pm 0.2$ & 0.39 \\
$P$ & 1-propanol & 60.1 & 1.58 & $12.9 \pm 0.3$ & 0.30 \\
$B$ & 1-butanol & 74.12 & 1.66 & $13.9 \pm 0.4$ & 0.39 \\
$P e$ & 1-pentanol & 88.15 & 1.64 & $15.0 \pm 0.4$ & 0.32 \\
$H$ & 1-hexanol & 102.17 & 1.42 & $16.7 \pm 0.3$ & 0.69 \\
$O$ & 1-octanol & 130.23 & 1.71 & $19.3 \pm 0.5$ & 1.82 \\
$W$ & water & 18.02 & 1.85 & $13.9 \pm 0.3$ & 0.30
\end{tabular}

subtracted from the SAXS curves and next curves were corrected taking into consideration both sample thickness and transmittance. The data were converted to absolute intensities with a calibrated Lupolen $\mathbb{R}$ ) (polyethylene) standard [15].

\section{Results and discussion}

The SAXS curves of investigated cellulose membranes are shown in Fig. 1 in double-logarithmic plots. It is clear that scattering intensity of the membranes coagulated in 1-hexanol and 1-octanol is much stronger than for other membranes. Because small-angle X-ray scattering is conditioned by the existence of the electron density inhomogeneities in the sample, which in the case of cellulose membranes is due to the existence of pores in them, the higher intensity is caused by higher content of the pores in these membranes.

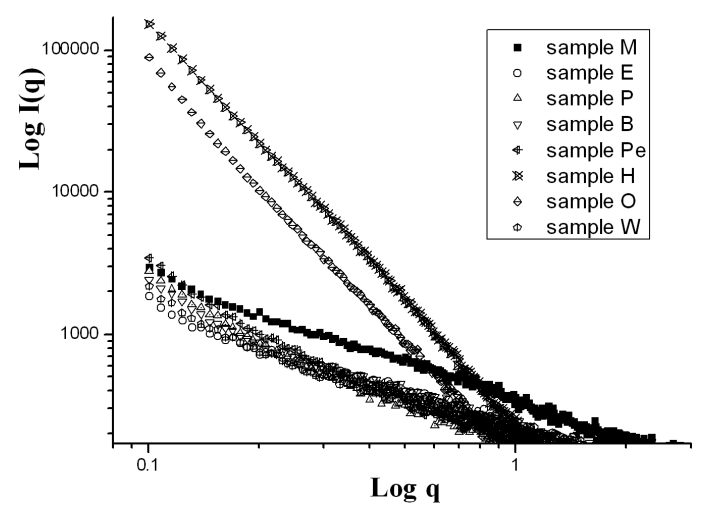

Fig. 1. Double logarithmic SAXS-curves of cellulose membranes.

Content of pores can be determined by calculating the so-called scattering power, or invariant $Q$, using the equation

$$
Q=\int_{0}^{\infty} q^{2} I(q) \mathrm{d} q=\varphi(1-\varphi)(\Delta \rho)^{2},
$$

where $I(q)$ is the corrected SAXS intensity, $\Phi$ is the volume fraction of pores and $\Delta \rho$ is the electron density difference between the pores and cellulose. The pores may be assumed to be entirely air filled in dry membranes. The electron density difference between cellulose and pores was calculated to be $\Delta \rho=511$ electrons $/ \mathrm{nm}^{3}$ [16]. Calculated by Eq. (1) values of $\Phi$ are presented in Table I.

For the determination of pore sizes the Guinier approximation, describing the scattering intensity for small values of the scattering vector $q$, can be used

$$
I(q)=I_{0} \exp \left(-\frac{q^{2} R_{\mathrm{G}}^{2}}{3}\right) .
$$

This equation describes the mean intensity of the radiation scattered by a particle of any shape, averaged through all its possible orientations in space. The parameter $R_{\mathrm{G}}$ is the electron radius of gyration, also called the Guinier radius. $R_{\mathrm{G}}$ can be determined from the slope of the plot of $\ln (I(q))$ versus $q^{2}$ using the intensity data in the low $q$ region. An example Guinier plot for a membrane $B$ is shown in Fig. 2 , and $R_{\mathrm{G}}$ values for all investigated membranes are given in Table $\mathrm{I}$.

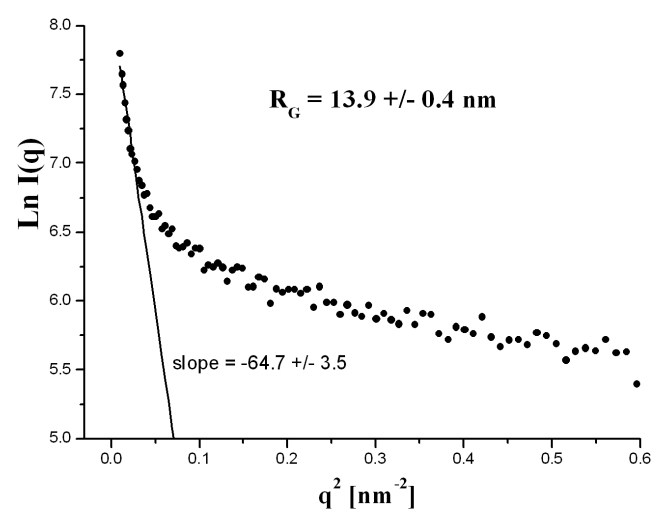

Fig. 2. The Guinier plot for the sample B.

Analysis of the results (Table I) indicates strong dependence of the Guinier radius of pores on the molar mass of coagulant used. Figure 3 shows that the $R_{\mathrm{G}}$ increases almost linearly with increasing molar mass of the organic coagulant. Water is an inorganic compound, so it is not surprising that in this case value of $R_{\mathrm{G}}$ does not correspond to this relationship (sample $W$ ). Water is the simplest and cheapest polar solvent and therefore has been used as a coagulant to obtain cellulose membranes. This coagulant has the lowest molar mass and largest dipole moment of all the other coagulants used in this work. The role of these two factors will be discussed later in this article. The volume fraction of pores in the studied membranes also depends on the coagulant molar mass. In Fig. 3 it can be seen that the pore content is almost the same in the range of molar mass from $18.02 \mathrm{~g} / \mathrm{mol}$ to $88.15 \mathrm{~g} / \mathrm{mol}$. The only exception is here 
the sample $M$, for which the $\Phi$ considerably exceeds the average value in that range. For membranes prepared using coagulants of the highest molecular mass, the content of the pores increases rapidly and for the membrane coagulated in 1-octanol is almost five times higher than in membranes obtained using coagulants of lower molar mass.

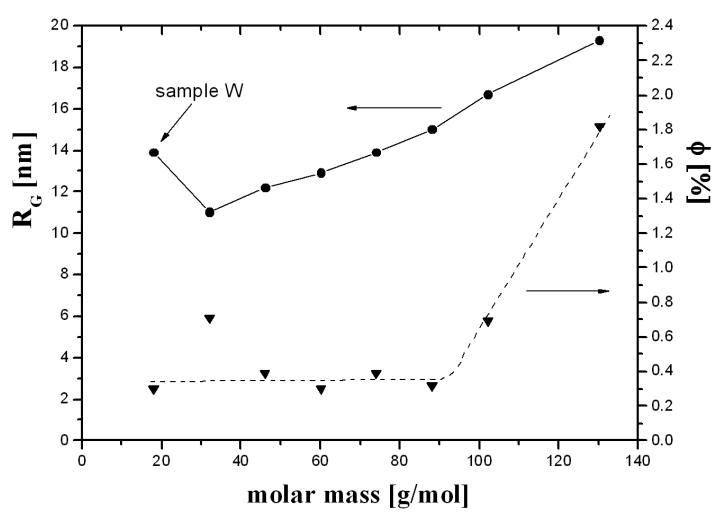

Fig. 3. The radius of gyration $\left(R_{\mathrm{G}}\right)$ and the volume fraction of pores $(\Phi)$ as a function of a coagulant molar mass.

To better understand the results obtained, the mechanism of solvation of cellulose at the microscopic level should be taken into account. The ionic liquid used to dissolve the cellulose is a chemical compound consisting of ion-bonded anion and cation. In the liquid state at room temperature the solvent molecules are dissociated. After mixing the cellulose and the ionic liquid these cations and anions attack the hydrogen bonds of cellulose, tearing them. Disruption of hydrogen bonds results in the separation of individual cellulose chains and allows them to entry into the solution. Regeneration of cellulose is based on precipitation of cellulose solution by the polar solvent (coagulant), which washes a ionic liquid, but does not dissolve cellulose. Washing the ionic liquid results in the formation of hydrogen bonds between cellulose chains, resulting in recovery of the structure of regenerated cellulose. The process of removing the solvent molecules by the coagulant molecules leads to the formation of the porous structure of the resulting cellulose. Therefore, it appears that the molecular weight of the coagulant molecules has a significant impact on the size of the pores formed in this process.

Coagulation process, described above, is based mainly on the Coulomb interactions between molecules of the solvent and the coagulant. It therefore seems reasonable that this process will also depend on the value of the dipole moments of interacting molecules. Our research has shown that the dipole moment of the coagulant is the second factor determining the content of pores in membranes, in addition to the molar mass. However, the effect of both these factors is complex, as illustrated in the dependence of the content of pores as a function of dipole moment shown in Fig. 4. Undoubtedly, the

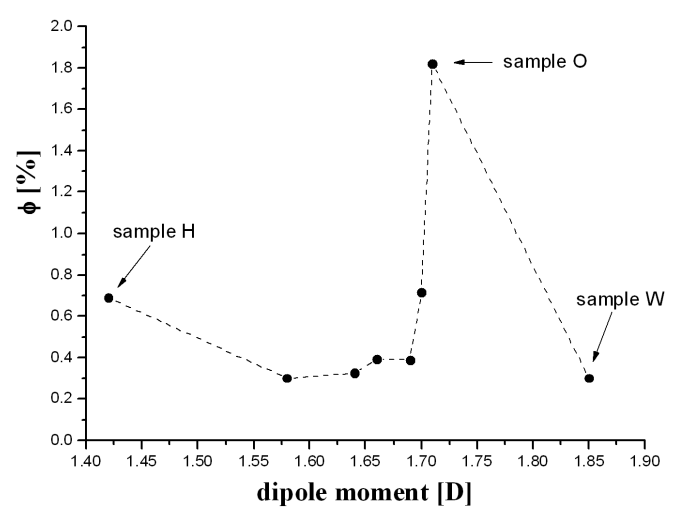

Fig. 4. The volume fraction of pores $(\Phi)$ as a function of a coagulant dipole moment.

content of the pores in the membrane is greatest when the value of the two factors is large, as in the case of sample $O$. If the dipole moment is large, and the molar mass is small, as in the case of water, the content of the pores is small. The same is true in the opposite case, when the molecular weight is relatively large, but the dipole moment is small, as in the case of the membrane $H$.

Summing up, discussed above studies have shown the influence both the molar mass and the dipole moment of coagulant on the content and size of the pores in the cellulose membranes. However, it is difficult to definitely determine which of these factors have a crucial impact on the number of pores in the membranes. It should be emphasized that, according to our knowledge, presented studies are the first attempt to establish at the molecular level the physical factors affecting these structural parameters of membranes that are important from the point of view of their applications.

\section{Conclusions}

The SAXS results show the existence of pores with a size of nanometer in the studied cellulose membranes. This study suggests that both content and dimensions of pores depend on molecular mass of the coagulant used. Also, it was found that the dipole moment of coagulant molecules has a large influence on the volume content of the pores. The highest content of pores is obtained when these two factors represent a substantial value. In the case where any of these factors is of little value pore content decreases significantly.

\section{Acknowledgments}

The present work was performed within the framework of the project titled "Biodegradable fibrous products" upon Contract No.: POIG.01.03.01-00-007-/08.

\section{References}

[1] X.-L. Li, L.-P. Zhu, B.-K. Zhu, Y.-Y. Xu, Sep. Purif. Technol. 83, 66 (2011).

[2] H.-P. Fink, P. Weigel, H.J. Purz, J. Ganster, Prog. Polym. Sci. 26, 1473 (2001). 
[3] K.J. Edgar, Ch.M. Buchanan, J.S. Debenham, P.A. Rundquist, B.D. Seiler, M.C. Shelton, D. Tindall, Prog. Polym. Sci. 26, 1605 (2001).

[4] R. Bodvic, A. Dedinaite, L. Karlson, M. Bergström, P. Bäverbäck, J.S. Pedersen, K. Edwards, G. Karlsson, I. Varga, P.M. Claesson, Coll. Surf. A Physicochem. Eng. Asp. 354, 162 (2010).

[5] H. Jin, Z. Chunxi, G. Lixia, Carbohydr. Res. 342, 851 (2007).

[6] N. Gathergood, M.T. García, P.J. Scammells, Green Chem. 6, 166 (2004).

[7] B. Jastorff, R. Störmann, J. Ranke, K. Mölter, F. Stock, B. Oberheitmann, W. Hoffmann, J. Hoffmann, M. Nüchter, B. Ondruschka, J. Filser, Green Chem. 5, 136 (2003).

[8] M.J. Earle, K.R. Seddon, Pure Appl. Chem. 72, 1391 (2000).

[9] N.P. Novoselov, E.S. Sashina, O.G. Kuz'mina, S.V. Troshenkova, Russ. J. Gen. Chem. 77, 1395 (2007).
[10] A. Pinkert, K.N. Marsh, S. Pang, M.P. Staiger, Chem. Rev. 109, 6712 (2009).

[11] S. Zhu, Y. Wu, Q. Chen, Z. Yu, C. Wang, S. Jin, Y. Ding, G. Wu, Green Chem. 8, 325 (2006).

[12] D. Ruan, L. Zhang, Y. Mao, M. Zeng, X. Li, J. Membr. Sci. 241, 265 (2004).

[13] C.R. Rambo, D.O.S. Recouvreux, C.A. Carminatti, A.K. Pitlovanciv, R.V. Antônio, L.M. Porto, Mater. Sci. Eng. C 28, 549 (2008).

[14] H. Stabinger, O. Kratky, Makromol. Chem. 179, 1655 (1978).

[15] O. Kratky, I. Pilz, P.J. Schmitz, J. Coll. Interf. Sci. 21, 24 (1966).

[16] J. Crawshaw, R.E. Cameron, Polymer 41, 4691 (2000). 